\title{
Circuit
}

Musiques contemporaines

\section{Établir les contacts}

\section{Réjean Beaucage}

Volume 13, numéro 3, 2003

Électroacoustique : nouvelles utopies

URI : https://id.erudit.org/iderudit/902288ar

DOI : https://doi.org/10.7202/902288ar

Aller au sommaire du numéro

Éditeur(s)

Les Presses de l'Université de Montréal

ISSN

1183-1693 (imprimé)

1488-9692 (numérique)

Découvrir la revue

Citer cet article

Beaucage, R. (2003). Établir les contacts. Circuit, 13(3), 85-96.

https://doi.org/10.7202/902288ar

Ce document est protégé par la loi sur le droit d'auteur. L'utilisation des services d'Érudit (y compris la reproduction) est assujettie à sa politique d'utilisation que vous pouvez consulter en ligne.

https://apropos.erudit.org/fr/usagers/politique-dutilisation/
Cet article est diffusé et préservé par Érudit.

Érudit est un consortium interuniversitaire sans but lucratif composé de l’Université de Montréal, l'Université Laval et l'Université du Québec à Montréal. Il a pour mission la promotion et la valorisation de la recherche. https://www.erudit.org/fr/ 


\title{
Établir les contacts
}

\author{
Réjean Beaucage
}

Je ne veux même pas savoir s'il y a eu des hommes avant moi.

Cette citation attribuée à René Descartes, imprimée en 1919 en travers de la couverture de la revue Dada $3^{\prime}$, signalait la première des tabula rasa que les différents mouvements artistiques et courants de pensée allaient accumuler tout au long du $x x^{e}$ siècle. II semble cependant que bon nombre d'artistes et d'observateurs du début du XXI ${ }^{e}$ soient plutôt enclins à rechercher des ascendances plutôt qu'à couper les ponts. Nous allons jeter un coup d'œil à trois productions parues en 2002 qui s'inscrivent dans ce mouvement: le livre Techno rebelle - un siècle de musiques électroniques ${ }^{2}$, et les disques An Anthology of Noise \& Electronic Music/First a-chronology volume $1^{3}$ et lannis Xenakis: Persepolis - Remixes Edition $1^{4}$.
1. Repris dans SANOUILLET, Michel et Dominique Baudouin, Dada - réimpression intégrale et dossier critique de la revue publiée de 1917 à 1922 par Tristan Tzara, tome 1, Centre du xxe siècle, Nice, 1976.

2. D'Ariel Kyrou, publié chez Denoël dans la collection «X-trême " (Paris, 2002, 428 pages).

3. Publié par l'étiquette belge Sub Rosa (SR 190).

4. Publié par l'étiquette américaine Asphodel (ASP 2005).

\section{Techno rebelle... et redondante}

Le livre Techno rebelle - un siècle de musiques électroniques établit des liens de filiation entre les diverses avant-gardes musicales du $x x^{e}$ siècle. Prenant parti pour les processus de création qui ont recours au ludisme et au hasard contre ceux qui se basent sur la recherche scientifique, l'auteur choisit la part de la folie contre la raison, cherchant dans la foulée à donner un vernis de respectabilité aux expériences et découvertes faites par les artistes des différents courants de la musique dite populaire. Ainsi, comme on peut le lire en quatrième de couverture:

Ariel Kyrou a choisi d'éclairer les filiations entre la scène électronique actuelle et ses innombrables ancêtres. Se croisent et se décroisent ainsi le dub et la musique contemporaine, le mouvement dada et les illuminés du rock allemand, le jazz libre et le hip hop new-yorkais, les agités de l'électro-pop et les fondus de la house, les pionniers de l'électricité et les siphonnés du sampling, les musiques de club et les musiques expérimentales, les futuristes italiens du siècle passé et les bébés bruitistes des free parties du temps présent. 
L'auteur y enfonce essentiellement le clou de la dichotomie entre musique institutionnelle et musique vernaculaire. Les "ancêtres " et les "pionniers " sont dans le premier camp, tandis que les «illuminés》, "agités", "fondus "et "siphonnés», sont dans l'autre. Techno rebelle se présente comme "un manifeste autant qu'un livre de référence ${ }^{5}$ » et à ce titre, l'auteur y soigne le côté manifeste en prenant, bien entendu, le parti des bons contre celui des méchants. II affine son discours dès le début du livre dans un chapitre intitulé "Pour comprendre mes partis pris». On m'excusera d'en citer ici un long extrait, mais il me semble éclairer admirablement le propos du livre:

Pour sauter de Marinetti à Daft Punk, j'ai suivi ma mauvaise foi avec le maximum d'honnêteté, sans égard pour les morpions du marché, les chèvres des hitparades et les sycophantes de l'industrie du divertissement. Je n'ai pas voulu pondre une encyclopédie, mais un hymne aux accidents de la création. Un chant de résistance. Une farce sérieuse et augmentée. Intellectuel gourmand plutôt que danseur impénitent, j'ai fait des choix subjectifs, construisant ma propre échelle de références sans écouter les sirènes de la renommée ou les inscriptions aux panthéons du disque. [...] Sur un autre territoire, plus polémique, j'assume l'anarchie de John Cage contre la science de Pierre Boulez. Le premier ne se voulait pas professeur, mais il a transmis ses virus de hasard aux artistes que i'aime et qui hantent ce livre, de Brian Eno à Holger Czukay, là où le second, enseignant talentueux, s'est fait le héraut de l'Art "véritable», ne livrant son savoir qu'à des étudiants ou des artistes patentés. [...] Boulez, grand prêtre du sérialisme intégral, a fermé les chants du possible, au bonheur d'une musique contemporaine qui s'en est transformée parfois en secte d'élus ascétiques.

Certains cherchent encore à préserver cette secte dans son intégrité illusoire. Pour ces fanatiques, heureusement en voie de disparition, les musiques populaires ou issues de ce terreau n'ont d'objectifs que "d'ordre commercial et statistique", cultivent toutes un "rythme obstinément binaire " et se cantonnent "à un rôle collectif, d'uniformisation". "

Le moins que l'on puisse dire à propos de l'«hymne aux accidents de la création » d'Ariel Kyrou est bien qu'il n'est pas un modèle de livre de référence. Les citations proviennent de livrets ou pochettes de disques, d'entrevues publiées dans des revues plus ou moins obscures, de "morceau(x) d'histoire ", "reportage devenu classique " ef autres "article(s) d'anthologie " souvent rédigés par des amis, voire de sites Internet, plusieurs de ceux-ci étant déjà disparus au moment d'écrire ces lignes. L'auteur fut rédacteur en chef adjoint de la "très branchée » revue Actuel (1989-1993), fondateur il y a une quinzaine d'années d'une entreprise du nom de Moderne Multimédias, et il est toujours chroniqueur, à France Culture, de l'émission Net + ultra. Cette émission s'intéresse à la "cyberculture", "cette culture multiforme qui naît d'Internet, des outils multimédias et des artistes électroniques ${ }^{7}$ ", et est produite par Jean-Philippe Renoult, qui signe ici la postface. Ce dernier coécrivait en 1999 un livre intitulé Global Tekno, dont Techno Rebelle est largement inspiré.

Le problème principal du livre est que son auteur a voulu tout y mettre, de dada à Zappa et du futurisme au disco. Bien sûr, avec les raccourcis nécessaires, tout peut
5. Encore la quatrième de couverture.

6. p. 23-24 - Les citations renvoient à un texte de Daniel D'Adamo inclus dans l'ouvrage collectif L'art au risque de la technique, Éditions Complexe, 2001. Sans être mensongère, la prose de $M$. Kyrou pourrait faire oublier au lecteur que Brian Eno a tout de même fréquenté la lpswitch Art School et la Winchester School of Art, avant de se joindre au Scratch Orchestra de Cornelius Cardew et à la Portsmouth Sinfonia à titre de clarinettiste ; que Holger Czuckay a suivi l'enseignement de Karlheinz Stockhausen pendant trois ans; que John Cage s'est très probablement fait appeler Professor quelques fois lorsqu'il occupait ce poste à la New School for Social Research de New York, à la University of South Florida ou même à Harvard ; et que Pierre Boulez, "enseignant talentueux", a déjà déclaré "[...] je ne suis pas très doué pour l'enseignement [...] je ne $m^{\prime}$ intéresse pas fondamentalement à la pédagogie parce que je n'y crois pas. Je crois au hasard " (dans un entretien avec Claude Samuel publié dans Éclats/Boulez, Éditions du Centre Pompidou, Paris, 1986, p. 111 .

7. Citation prise, eh oui... sur le site Internet de l'émission :

http://www.radiofrance.fr/chaines/franceculture2/netultra/presentation.php 
éventuellement être relié, et si beaucoup des liens que fait l'auteur peuvent en effet être légitimés, ce ne sera pas par ses explications réductrices, qui induisent le lecteur en erreur assez souvent. Écrit à la sauce impressionniste du "nouveau journalisme», le livre de Kyrou se lit parfois comme un poème tant l'auteur aime l'usage des images fortes. Ainsi, "la première représentation du Sacre du printemps d'lgor Stravinsky tourne au massacre » (p. 36), Érik Satie est un "vague suppôt de dada» (p.36), et Brian Eno " un artiste se défiant des artistes; un chasseur de hasards; I'humain de la machine; un musicien sans codes à penser; un peintre des sons; un chercheur de textures sonores et visuelles; un chercheur d'Afrique ; [... un cuisinier émérite ; un père de famille tout aussi honorable; le seul penseur adapté à l'ère cyber-technoïde; le géniteur de la musique ambient; un héritier de Jonathan Swift et de Marcel Duchamp et au final rien de tout cela... » (p. 340). Ce style alambiqué devient par ailleurs assez vite lassant.

Un autre reproche que l'on peut lui faire est sa propension à infantiliser le travail des créateurs. À le croire, toute la musique du $x x^{e}$ siècle ne serait basée que sur une suite de hasards et d'accidents engendrés par des artistes lancés dans des démarches expérimentales pour le seul ludisme de la chose. Ainsi, entre autres exemples, les membres du duo électro Autechre, lorsqu'ils composent, "ne pensent pas [...] ne cherchent pas. Ils s'amusent... » (p. 313), William Burroughs invente "par hasard la technique du cut-up" (p. 249) et celle-ci encore, qui donne la mesure de ce que l'auteur pense de la musique contemporaine:

Edgar Varèse, Karlheinz Stockhausen, Pierre Schaeffer, Olivier Messiaen ou Pierre Henry dérangeaient l'honnête homme avec leurs tribulations électroniques, concrètes ou bruitistes, mais ils n'avaient pas besoin de remettre en cause la hiérarchie entre le technicien et le musicien, le colleur de sons et le compositeur de musiques. Ils étaient à la fois techniciens et musiciens, manipulateurs et compositeurs. Et donc, au final, on ne retenait de leurs leçons qu'un nouvel art de la composition. Seule une élite cultivée allait au-delà des apparences, appréciant les délices esthétiques de ces rebelles géniaux, rangés par commodité dans les placards de la musique contemporaine comme pour apposer l'étiquette rassurante de la recherche savante sur leurs œuvres. Le plaisir, voyez-vous, cela fait sale (p. 174).

Le voilà prouvé une bonne fois pour toute : il est impossible de s'amuser en faisant de la recherche! On plaint les générations de créateurs qui n'ont pas eu la bonne idée de laisser travailler le hasard.

En bref, l'auteur est obnubilé par certains faits tirés de l'histoire récente et qui lui font voir l'histoire au travers d'un filtre brouillé : oui, il est de plus en plus possible, grâce aux développements de l'informatique, de faire de la musique sans être musicien, cela ne signifie pas que tous ceux qui le font soient des génies et leurs musiques, des chef-d'œuvres qui repoussent chaque fois plus loin les limites de l'imagination. Oui, le mouvement punk et ses dérivés ont revendiqué le droit de faire de la musique après deux minutes d'apprentissage des règles de l'art, mais cela ne veut pas dire qu'aucun des artistes de ces mouvances n'ait cherché à pousser plus loin 
ses recherches, réalisant peutêtre ainsi les pièces que l'on juge encore intéressantes aujourd'hui. II n'est pas inutile non plus de considérer que beaucoup d'artistes "pop " ont traversé l'histoire pour bien autre chose que leur musique, les lois de la mise en marché n'y étant pas toujours étrangères... Eh ovi, John Cage a beaucoup théorisé sur l'utilisation du hasard en composition et Pierre Schaeffer a "découvert» la musique concrète par "accident». N'empêche, lorsqu'il l'a découverte, il l'a quand même reconnue, et a délibérément choisi de travailler la question... Et le hasard n'est pas tombé dans l'art musical de John Cage comme la pomme sur la tête de Newton, mais à la suite d'une longue réflexion visant à renouveler, entre autres choses, les rapports entre interprète et compositeur. Bien sûr, le hasard et les accidents de parcours ont leur importance dans I'histoire de l'art comme dans celle de I'humanité, mais tout n'est pas si simple et l'on peut se demander s'il est vraiment utile de remonter à Luigi Russolo pour tracer le génotype de la musique acid-house...

Ce que l'on retiendra surtout du livre de Kyrou, ce sont des pistes et quelques références (quand on peut les retrouver). Parmi ces dernières, le projet Modulations, de la compagnie américaine d'arts et cultures multimédias Caipirinha, dirigée par la cinéaste Lara Lee. Celle-ci a fait paraître en 1998 le film Modulations - Cinema for the Ears, qui suivait exactement le même parcours que celui qu'empruntera Kyrou 4 ans plus tard. Deux disques compacts ${ }^{8}$ ont également été édités dans le cadre de ce projet et, en 2000, un livre réunissant, sous la direction de Peter Shapiro, une pléiade de spécialistes des différents sous-genres de musique électronique. On trouve aussi à la base du travail de Kyrou le livre de David Toop Ocean of Sound: Aether Talk, Ambient Sound \& Imaginary Worlds ${ }^{9}$, qui est aussi accompagné d'une compilation publiée sur double disque compact sous étiquette Virgin. Bref, il sera sans doute préférable de se tourner vers les références, plutôt que vers le patchwork mal dégrossi qu'en a régurgité Kyrou.
8. Modulations - Cinema for the Ears 1998, Caipirinha music (CAl2018) et Early Modulations: Vintage Volts, 2000, Caipirinha music (CAI.2027.2.)

9. Version originale publiée chez Serpent's Tail, Londres (1995, 224 p.). Kyrou, qui parle candidement $d$ ' " une influence forte", se réfère cependant à la traduction 10 cean of Sound, Ambient Music, Mondes imaginaires et voix de l'étherl parue chez Kargo/l'Éclat en 2000.

\section{À la source}

L'étiquette Sub Rosa a été fondée à la fin des années 1980 en Belgique par Frederic Walheer et Guy Marc Hinant, afin de fournir un support à certains créateurs marginaux et de rendre disponibles des documents d'archives rares et importants. C'est ainsi qu'on trouve au catalogue de l'étiquette des documents sonores qui nous permettent de réentendre les voix de Guillaume Apollinaire, Tristan Tzara, Marcel Duchamp, William S. Burroughs ou James Joyce, mais aussi des œuvres d'Henri Pousseur, de Nam June Paik, Morton Feldman ou Konrad Boehmer. À ces archives s'ajoutent des productions récentes de compositeurs plus près de la musique actuelle ou des nouveaux courants électros comme Fred Frith, Bill Laswell, Robin Rimbaud ou David Shea. Un catalogue ambitieux donc qui, comme le livre de 
Kyrou, couvre le siècle dans le but évident de fournir des bases aux créateurs contemporains $^{10}$.

Les directeurs de l'étiquette Sub Rosa sont grandement influencés par la pensée du philosophe Gilles Deleuze et en particulier par le concept de "rhizome" " , défini dans l'ouvrage qu'il a rédigé avec le psychiatre Félix Guattari, Milles Plateaux ${ }^{12}$. Ainsi Guy Marc Hinant écritil, dans les notes préliminaires du livret accompagnant le disque An Anthology of Noise \& Electronic Music / First a-chronology volume 1 :

Our method is not purely historical [...]. By drawing a line - curved not straight and by choosing a specific aspect (e.g. the concept of noise in music), we can establish links between more or less well-known artists le.g. between the inventor of concrete music Pierre Schaeffer and the Italian futurist Luigi Russolo, author of The Art of Noises in 1913, who at the time was regarded as a pleasant curiosity but whose approach and indirect heritage seem to us more relevant than some "real composer " whose prestige was then uncontested). History needs constant re-evaluation because, like music, history cannot be read as a fixed entity.

Sub Rosa prefers to regard things as strata. [...] The music that we are concerned with, some of which was never published, nor even heard, in its time, require us to rethink categories such as "serious" music in the learned tradition - the plateau Bach-MozartSchönberg to which we prefer rhizomes such as the duo Duchamp-Cage or the configurations Varèse-Xenakis-lkeda-Boehmer and Hampson-Pousseur-Oval, or even Schumann-Feldman-Cage ${ }^{13}$.

L'anthologie vise donc, avant tout, à rendre disponible des documents dont les auteurs sont interconnectés par un réseau de liens rhizomatiques qui traverse le temps, la "quête étant embellie par l'impossibilité de tout inclure ${ }^{14}$ ", ce dernier point n'empêchant pas la sélection d'être des plus intéressantes. Débutant par une séquence chronologique, le premier disque s'ouvre sur Corale (1921), de Luigi et Antonio Russolo, pour orchestre et intonarumori ${ }^{15}$, une œuvre vraiment mineure, mais grâce à laquelle les compositeurs ouvraient pourtant des sentiers nouveaux dans lesquels allaient s'engouffrer par la suite des hordes de chasseurs de bruits. Vient ensuite l'œuvre d'un autre pionnier qui, en produisant le premier film sans images, allait ouvrir les voies parallèles de la musique concrète et du cinéma pour l'oreille à de nombreux électroacousticiens à venir. II s'agit de Wochende (1929-30) du cinéaste Walter Ruttman qui, en choisissant de n'utiliser que la portion sonore de la pellicule, laissant son public affronter un écran blanc, invente le montage sonore et confère au son une autre valeur que celle qui lui est habituellement attribuée. Ce qui, incidemment, nous ramène à Gilles Deleuze, qui, dans Cinéma 2 : L'image-temps, "analyse le processus par lequel le son en est venu à rompre les chaines qui l'attachaient au visuel. Selon lui, le son se serait libéré de l'image et serait devenu une image en soi : une image-son, qui se distingue radicalement de l'image visuelle ${ }^{16}$, .

Comme on le sait, Pierre Schaeffer allait systématiser sous l'appellation « musique concrète" ce qui chez Ruttman n'était encore qu'une intuition géniale. Le premier
10. Extrait de la notice du disque Sonology Institute - Electronic Music 1959-1969 (SR 164) [je traduis] : «Nous devons nous pencher sur cette période - celle de la révolution des années 1950 et 1960 - et comprendre que ce qui était élaboré à cette époque, l'était sans base - ex nihilo - une musique produite par des machines destinées à construire la nouveauté. "

11. Extrait de Mille Plateaux: "Résumons les caractères principaux d'un rhizome : à la différence des arbres ou de leurs racines, le rhizome connecte un point quelconque avec un autre point quelconque, et chacun de ses traits ne renvoie pas nécessairement à des traits de même nature, il met en jeu des régimes de signes très différents et même des états de non-signes. Le rhizome ne se laisse ramener ni à l'Un ni au multiple. II n'est pas l'Un qui devient deux, ni même qui deviendrait directement trois, quatre ou cinq, etc. [...] II n'est pas fait d'unités, mais de dimensions, ou plutôt de directions mouvantes. "

12. Les Éditions de minuit, 1980.

13. Traduction de l'auteur: Notre méthode n'est pas purement historique. En traçant une ligne - courbe - et en choisissant un aspect spécifique (par exemple le concept de bruit en musiquel, on peut établir des liens entre des artistes plus ou moins bien connus (par exemple, entre l'inventeur de la musique concrète Pierre Schaeffer et le futuriste italien Luigi Russolo, auteur de L'art des bruits en 1913, dont les théories étaient à l'époque considérées comme des curiosités amusantes, mais dont l'approche et l'héritage indirecte nous apparaissent plus substantiels que ceux de certains "vrais compositeurs" dont le prestige était alors incontestable). L'histoire doit constamment être réévaluée parce que, comme la musique, elle ne peut être considérée comme un objet fini.

Sub Rosa préfère voir les choses par strates. Les musiques qui nous intéressent, dont certaines ne furent jamais publiées, ou même entendues (à leur époque) nous portent à réexaminer des catégories comme celle de la "musique sérieuse "traditionnelle - le plateau Bach-Mozart-Schönberg auquel nous préférons des rhizomes comme le duo Duchamp-Cage ou les configurations Varèse 
sillon qui se mange la queve, répétant inlassablement le même fragment abstrait, la musique qui tient dans la main plutôt que sur le papier, c'est Schaeffer qui, le premier, en approfondit la recherche. De ses Cinq études de bruits (1948), c'est l'Étude violette que l'on a retenue ici. Premiers rythmes bouclés, premières attaques renversées, les sons, en devenant concrets, perdent pourtant leurs contours clairs et l'on n'est plus toujours certains de bien entendre ce que l'on croit entendre. Ici, vraiment, un nouveau monde s'ouvrait. Puis, en 1953 aux studios de la WDR à Cologne, un autre, encore. Henri Pousseur y rejoignait Karlheinz Stockhausen en 1954 et réalisait une première étude de musique électronique sous le titre Séismogrammes. C'est cependant en 1957, au Studio di Fonologia que dirigeait Luciano Berio à Milan, qu'il réalisait Scambi, qui est incluse ici. Pousseur, Stockhausen ou Boulez avaient été attirés vers l'appareillage électronique par la possibilité qu'il semblait pouvoir offrir d'un contrôle total sur le matériau musical. Tout n'allait pas être si simple...

Ainsi, je partais d'une seule source, le "bruit blanc "et, par des méthodes de sélection de différentes stratifications /méthodes qu'il serait trop long de décrire ici, mais dont je peux principalement dire qu'elles contenaient toujours un certain degré d'imprévisibilitél, je produisis à partir de ce "magma " de base un matériau ayant le caractère bruiteux et irrégulier que je souhaitais, tout en me permettant aussi de le contrôler d'une manière efficace. Il s'agissait d'un contrôle global ne concernant pas chaque élément, mais des groupes entiers d'éléments : je pouvais déterminer globalement le mouvement de hauteur et la vitesse statistique des impulsions, éléments brefs que j'avais extraits du bruit blanc. Je pouvais aussi contrôler la transformation graduelle de ces éléments et groupes d'éléments en surfaces continues, grises et mobiles, ainsi que leurs interruptions par des silences plus ou moins précis, de longueurs et en nombres variables. Je ne composais pas une pièce achevée. Sous le titre Scambi (Échanges), je fis un certain nombre de séquences séparées, qui pouvaient être combinées dans la succession et aussi dans la superposition d'après certaines règles de connexion bien définies. Je fis personnellement deux versions différentes, et quelques autres musiciens, tel Berio, utilisèrent cette possibilité $d^{\prime}$ exercice rapide et réalisèrent leurs propres versions ${ }^{17}$.

Ce faisant, l'air de rien, Henri Pousseur est probablement devenu le premier compositeur dont la musique a été remixée! Impossible de savoir laquelle de ses deux versions est reproduite ici, ni, malheureusement, d'en modifier l'ordre des séquences comme le voudrait le principe de l'œuvre ouverte qui a présidé à sa conception, mais le résultat est, de toute façon, magistral et donne la mesure des possibles qui s'offraient encore au compositeur à cette époque.

Le parcours chronologique se termine par un premier voyage en Amérique pour découvrir la musique de Gordon Mumma. Après des études de piano et de trompette, et un début de carrière de trompettiste au sein de différents orchestres, il est cofondateur, en 1953, du Cooperative Studio for Electronic Music de Ann Arbor. II est, de 1966 à 1974, avec John Cage et David Tudor, l'un des trois compositeursinterprètes de la troupe de danse de Merce Cunningham. On comprend que son approche du phénomène musical est assez éloignée des expériences sérielles de
Xenakis-lkeda-Boehmer et Hampson-PousseurOval, ou même Schumann-Feldman-Cage.

14. Toujours le livret et une traduction de l'auteur.

15."[...] Le hululeur qui grogne, le grondeur qui barrit, le crépiteur qui stridule, le strideur qui miaule, le bourdonneur qui sifflote, le glouglouteur qui hoquette, l'éclateur qui mugit, le sibilleur qui émet de profonds rots, le croasseur qui vrombit, le froufrouteur qui chuinte, tous ces «instruments » dont aucun ne répond à sa dénomination et dont l'ensemble fait le pauvre bruit unique et monotone d'une scierie à eau dans la montagne, ne provoquent ni stupeur, ni crainte, ni scandale [...]". Extrait de la critique de Pierre Seize d'un concert donné à la galerie Montaigne le 17 juin 1921, publiée dans Bonsoir le 20 juin 1921 et reprise dans SANOUILLET, Michel, Dada à Paris tome 1, Nice, Centre du Xx siècle, 1980, p. 284.

16. Cité par Bernard Schültze, dans son article "Mycologie urbaine: Montréal, ses scènes et ses sons », dans Parachute $n^{\circ}$ 107, Montréal, juillet-août-septembre 2002, p. 109.

17. Pousseur, Henri, "Calcul et imagination dans la musique électronique ", dans Musique en jeu $n^{\circ} 1$, Seuil, Paris, 1970 , p. 37-38. 
Stockhausen et consorts. Le matériau est beaucoup plus brut que chez Pousseur, par exemple, et les sons électroniques côtoient ici les sons concrets, mais les choses ont déjà bien changée depuis Scambi, l'œuvre reproduite ici (The Dresden Interleaf 13 February 1945) datant de 1965.

C'est après cette pièce de Mumma que commence le parcours achronologique de l'anthologie et très bizarrement, on a choisi de le faire débuter par une autre pièce américaine datant de la même année que la précédente. Un choix d'autant plus difficile à justifier qu'il ne s'agit ni d'une œuvre de musique électronique ni, à proprement parler, de noise music, la qualité "bruitiste " du document étant visiblement due à de mauvaises conditions d'enregistrement. Le court extrait de la pièce Transe \#2 de Tony Conrad, Angus Maclise et John Cale n'est pas mauvais en soi et l'importance du rôle de chacun des membres de ce trio dans le développement de la musique américaine est indéniable ${ }^{18}$, mais l'œuvre instrumentale d'inspiration orientale ne semble tout simplement pas à sa place sur ce disque. Ce qui n'est pas le cas de la pièce suivante, enregistrée en concert à Bruxelles en 2000 par I'Anglais Philip Jeck, le Japonais Otomo Yoshihide et le Québécois Martin Tétreault. Le tourne-disque a fait du chemin depuis les premières découvertes de Pierre Schaeffer et ces trois platinistes ${ }^{19}$ en utilisent vraiment toutes les ressources. Sur Untiled \# $7^{20}$, reproduite ici, Jeck utilise des citations, modifiées ou non, Yoshihide manipule divers effets électroniques reliés à ses platines et Tétreault n'utilise que les différentes parties constituantes de ses platines (c'est-à-dire qu'il n'utilise aucun disque). Les trois instrumentistes sont rompus aux techniques de l'improvisation et la grande variété de textures qu' offre leur instrumentarium leur permet une exploration sonore qui donne la mesure du chemin parcouru. Plus de boucles ici, mais une construction complexe où les sons se répondent et se superposent avec une subtilité étonnante.

Luigi Russolo écrivait en 1913 dans son manifeste L'art des bruits ${ }^{21}$ :

Traversons ensemble une grande capitale moderne, les oreilles plus attentives que les yeux, et nous varierons les plaisirs de notre sensibilité en distinguant les glouglous d'eau, d'air et de gaz dans les tuyaux métalliques, les borborygmes et les râles des moteurs qui respirent avec une animalité indiscutable, la palpitation des soupapes, le va-et-vient des pistons, les cris stridents des scies mécaniques, les bonds sonores des tramways sur les rails, le claquement des fouets, le clapotement des drapeaux. Nous nous amuserons à orchestrer idéalement les portes à coulisses des magasins, le brouhaha des foules, les tintamarres différents des gares, des forges, des filatures, des imprimeries, des usines électriques et des chemins de fer souterrains.

L'auteur de ces lignes aurait sans doute apprécié de pouvoir assister à l'une des manifestations que les Américains Mark Pauline et GX Jupitter-Larsen organisent sous le nom de Surival Research Laboratories (SRL). Nécessitant généralement de très grands espaces, les "concerts " de SRL ressemblent davantage à des scènes de guerre moderne avec incendies, explosions et vacarme tonitruant qu'à une quelconque activité musicale. Pourtant, il s'agit bien de produire des sons et, éventuellement, de les enregistrer. L'œuvre reproduite ici, October 24, 1992 Graz, Austria,
18. Les trois ont été membre du Dream Syndicate de LaMonte Young, dont l'apport au courant américain de musique minimaliste est important ; Maclise et Cale sont deux des membres fondateurs, en 1965, de la formation de rock exploratoire Velvet Underground.

19. Le terme "platiniste » désigne celui qui se sert, comme instrument, d'une platine (Petit Robert: Élément d'une chaîne de reproduction sonore servant à la lecture des disques [...]) et a une connotation qualitative qui le différencie du DJ (Deejay, disc-jockey). Le DJ utilise généralement les disques pour faire danser ses auditeurs, tandis que le platiniste le fait pour un résultat plus abstrait.

20. Extrait d'une pièce improvisée en concert lors du festival Klinkende Munt de Bruxelles, le 14 juillet 2000. La pièce complète (27 minutes, 5 secondes) est parue sur le premier volume de la collection «Invisible Architecture», publiée par Audiosphere (ASO 1).

21. L'âge d'Homme, Lausanne, 1975. 
fut enregistrée lors d'un événement intitulé The Deliberate Evolution of a War Zone : A Parable of Spontaneous Structural Disintegration ${ }^{22}$. L'équipement nécessaire à cette représentation, à peine dévié de son habituel usage militaire, était manipulé par 22 techniciens. Qu'il suffise pour donner une idée du résultat sonore de mentionner que la police de la ville de Graz fut inondée d'appels de citoyens qui croyaient que la ville était sous attaque lla situation entre Serbes et Croates, à quelques kilomètres seulement de la frontière autrichienne, semblait pouvoir exploser à tout moment)... II n'y a certainement aucune comparaison possible entre le fait d'assister à ce genre de manifestation et l'écoute de l'enregistrement qui en résulte. $\mathrm{Ce}$ dernier donne cependant raison à Russolo quant à l'extraordinaire potentiel, encore assez peu exploré, $d u$ «bruit ».

À l'autre bout du spectre bruitiste se trouvent des microsons, dont la manipulation peut produire un résultat tout aussi étonnant que celle des sons d'amplitude tellurique. Craquements, cliquetis et autres subtils froissements sont les ingrédients de la pièce Ragout: Küchen Rezept (1998) de l'ensemble allemand Einsturzende Neubauten, formé en 1980 par des membres du collectif d'artistes Die Geniale Dilletanten. Cette forme de microsound ${ }^{23}$ " instrumental », par opposition à son habituel pendant électronique, pourrait être la musique de chambre du courant noise, là où le SRL serait l'équivalent d'une superproduction wagnérienne.

Le premier disque se clôt avec Aspekt (1966), une musique électronique de Konrad Boehmer. Né en 1941, il étudia d'abord avec Gotffried Michael Koenig à Cologne, puis Boulez, Pousseur et Stockhausen à Darmstadt. l'œuvre a été réalisée au Studio de sonologie d'Utrecht, auquel il collabora de 1966 à 1968 et dont il deviendra directeur en 1989. La réflexion politique est à la base du travail de Boehmer et Aspekt, dédiée à un jeune Vietnamien assassiné en 1964, est une œeuvre radicale qui rend compte de la fureur du compositeur devant la tournure sociopolitique que les événements prenaient dans les années 1960.

Le deuxième disque poursuit l'achronologie avec un Hommage à John Cage (1958-1959) de Nam June Paik, artiste mieux connu aujourd'hui pour ses installations vidéographiques, mais qui est d'abord venu à l'avant-garde par la musique. Ses rencontres avec Stockhausen et Cage, vers 1956, coïncidèrent avec ses premières explorations de la musique électronique. II s'agit cependant ici d'une musique concrète faite du collage chaotique, par succession ou superposition, de divers enregistrements. On entend le son caractéristique de l'aiguille du tournedisque retirée abruptement du sillon, des variations de vitesses, des enregistrements lus à l'envers, etc. L'œuvre est très judicieusement suivie du Rozart Mix (1965) de John Cage, tel que dirigé par Rainer Riehn en 1972. L'œuvre, pour au moins 4 interprètes avec au moins 12 enregistreuses et 88 boucles de ruban magnétique de dimensions variées, est un véritable feu d'artifice construit à partir de citations empruntés à la radio, la télévision ou repiquées sur des disques. On ne s'étendra pas ici sur le cas de Johñ Cage, qui est évidemment un pionnier de l'utilisation des tourne-disques, radio et autres équipements électroniques en situation de concert (on
22. Plus de détails sont disponibles sur le site Internet du Survival Research Laboratories à l'adresse : http://www.srl.org

23. Voir la définition de ce terme dans I'article de Ned Bouhalassa « Electroniquoi? Chronique de la naissance d'une nouvelle constellation sonore», publié dans Circuit, vol. $13, n^{\circ} 1$. 
pense aux Imaginary Landscapes, mais aussi aux Williams Mix, Fontana Mix ou aux différentes Variations).

Sonic Youth est une formation issue de la No Wave du début des années 1980 à New York. L'important succès qu'a connu la formation après avoir laissé le circuif rock indépendant pour joindre les rangs de l'écurie Geffen Records en 1990 a en quelque sorte établi ses membres (Thurston Moore, Lee Ranaldo, Kim Gordon ef Steve Shelleyl en tant que "parrains" de la musique underground américaine. En 1996, le quatuor lançait l'étiquette Sonic Youth Records (SYR) qui allait leur permettre de rendre disponibles leurs projets plus expérimentaux, et donc moins attrayants pour Geffen Records. Parmi ceux-ci, la formation a fait paraître un disque intitulé Goodbye 20th Century, sur lequel sont interprétées des œuvres de John Cage, Cornelius Cardew, Takehisa Kosugi, George Maciunas, Pauline Oliveros, Yoko Ono, Steve Reich, Nicolas Slonimsky, James Tenney et Christian Wolff. L'œuvre présentée ici, Audience (1983), a été enregistrée à la fin d'un concert alors que les membres du groupe ont tourné leurs micros vers la salle et mixé les sons produits par le public. L'enregistrement est joué au ralenti; son seul véritable intérêt est d'être inédit. Ce détail attirera sans doute les amateurs de la formation qui découvriront là, c'est certain, une véritable boîte de Pandore.

Suivent deux œuvres charnières datées de 1958 : le Poème électronique d'Edgar Varèse et Concret PH de lannis Xenakis. Leur absence dans cette anthologie produite par une étiquette belge aurait évidemment été inexplicable puisqu'elles font non seulement partie du patrimoine constituant l'histoire de la musique électronique, mais aussi parce qu'elles ont été conçues pour être diffusées dans le pavillon Philips, lors de l'exposition universelle de Bruxelles de 1958. C'est d'ailleurs une vue de ce fameux pavillon qui orne le boîtier de la compilation. Des pièces bien différentes, certes, mais qui sont toutes deux l'œuvre de visionnaires qui n'ont pas fini d'inspirer ceux qui aspirent à marcher dans leurs pas. L'extrait suivant date de 2001 et s'intitule FTP $>$ Bundle / Conduit 23. On le doit à Paul D. Miller, alias DJ Spooky (That Subliminal Kid), écrivain et artiste polymorphe issu du côté le plus exploratoire de la scène hip hop et éventuellement collaborateur de... lannis Xenakis lavec le STX Ensemble), qu'il considère comme l'un des plus grands compositeurs de l'histoire de la musique. Il est aussi l'un des inventeurs du illbient, versant sombre de la musique ambient, dont l'œuvre présentée ici est un exemple.

On revient en 1966 pour un long exercice (30 minutes) intitulé A Little Noise in the System (Moog System), de Pauline Oliveros. Une pièce inédite à propos de laquelle la notice dit qu'elle est un parfait exemple du genre de musique que cette anthologie veut empêcher de sombrer dans l'oubli et qu'il est beaucoup plus aisé de l'écouter aujourd'hui que cela pouvait l'être à l'époque de sa création. L'œuvre, une construction qui explore les diverses qualités de bruit blanc, du murmure à l'insoutenable, est en effet bruyamment radicale et pourra sembler extrêmement surprenante aux auditeurs qui connaissent le travail plus récent de la grande prêtresse du Deep Listening $^{\odot}$. 
Après la plus longue pièce de l'anthologie vient la plus courte, One Minute (1997), de Ryoii lkeda. Un autre exercice, de concision celuità, qui donne un très bref aperçu du noise à la japonaise, un courant qui a de nombreux représentants que l'on peut sans doute s'attendre à trouver sur les prochains volumes de cette anthologie. On peut regretter le peu de temps accordé à lkeda, un artiste qui construit depuis son premier album en 1995 un corpus d'une grande originalité.

En définitive, Sub Rosa propose une compilation à laquelle on ne peut trouver que des qualités. Extraits rares ou même inédits, parcours didactique, qualité de la reproduction, tout concourt à faire de cette Anthology of Noise \& Electronic Music un objet important que chaque nouveau volume, si la qualité se maintient, rendra davantage incontournable.

\section{Remixer Xenakis}

L'étiquette américaine Asphodel rivalise d'éclectisme avec Sub Rosa et se côtoient à son catalogue aussi bien John Cage et Francis Dhomont, que DJ Spooky ou les Invisbl Skratch Piklz... Son fondateur est Naut Humon, directeur artistique du collectif Sound Traffic Control, qu'il a fondé en 1992 afin de poursuivre les expériences qu'il menait déjà au sein de la formation Rhythm \& Noise depuis la fin des années 1960. Les "concerts » organisés par cette formation étaient qualifiés de "mobilisations " et se tenaient fréquemment dans de très grands espaces extérieurs ${ }^{24}$, la spatialisation des événements sonores étant une des préoccupations principales de Naut Humon. Personnage influent de la communauté électronique internationale, c'est à lui que l'on doit, entre autres réalisations, la mutation en 1999 de la catégorie Computer Music en Digital Music lors des compétitions du festival Ars Electronica.

Humon a codirigé le projet de réédition de Persepolis de Xenakis avec Zbigniew Karkowski, compositeur de musique expérimentale et membre du trio Sensorband (avec Edwin van der Heide et Atau Tanaka). Persepolis est d'abord parue au début des années 1970 chez Philips dans la collection "Prospective $21^{\mathrm{e}}$ siècle»; en 2000, Fractal Records a rééditée l'œuvre en disque compact avec la mention "version complète de 55 minutes ". Cette version que nous propose Asphodel, mixée au GRM par Daniel Teruggi sous la supervision du compositeur peu de temps avant sa disparition, fait pourtant bien 60 minutes et pourra sans doute être considérée dorénavant comme la version définitive. Créée au Festival de Shiraz à Persépolis le 26 août 1971, l'œuvre est une commande pour la commémoration du $2500^{\circ}$ anniversaire de la fondation de la Perse. II s'agit d'un "polytope " (œuvre multimédia) incorporant éclairages complexes, procession au flambeau et diffusion de la bande huit pistes sur 59 haut-parleurs disséminés sur le site extérieur ${ }^{25}$. La musique concrète de Persepolis peut sans doute être qualifiée de bruitiste; les sons ne s'y laissent guère
24. Tout comme ceux de Survival Research Laboratories, évoqués plus tôt, ou, bien sûr, comme les polytopes de Xenakis.

25. On connaît aussi les polytopes de Montréal en 1967, de Cluny en 1972 et de Mycènes en 1978. 
identifier aisément, mais c'est la couleur du métal strident qui domine, traversant sporadiquement une nébuleuse de texłures en constante métamorphose qui ne laisse aucune place au silence. La réduction sur deux pistes ne fait souffrir aucun handicap sérieux au fantastique ballet de timbres imaginés par le compositeur. L'ensemble évoque un événement d'ampleur cataclysmique de l'ordre du Big Bang, avec tout ce que cela comporte de catastrophique.

On a bien déjà entendu des remixages d'œuvres de Steve Reich ou de Pierre Henry, mais l'idée de "remixer " une œuvre d'une telle densité semble tout de même de prime abord saugrenue. C'est d'ailleurs pourquoi, malgré le titre du disque, ce n'est pas tout à fait ce que nous présente le second disque. II s'agit en effet davantage d'un hommage à Xenakis et les directeurs du projet ont demandé à leurs invités de s'inspirer de son œuvre afin de la " recomposer " et d'établir, à trente ans de distance, un dialogue avec le travail du précurseur. Le résultat est certes surprenant. L'œuvre de Xenakis sert de point de départ, bien sûr, mais chaque artiste y imprime ses préoccupations, sa manière, son rythme. Persepolis devient une partition conceptuelle par laquelle chacun produit un objet qui s'en inspire ou qui en explore un angle particulier. On regrette cependant que les notes ne nous apprennent rien sur les procédés utilisés par les artistes.

II serait un peu fastidieux de décrire par le détail chacune des contributions que compte le deuxième disque, mais quelques-unes valent tout de même d'être soulignées. Le compositeur et multi-instrumentiste japonais Otomo Yoshihide livre sans doute la version la plus proche de l'originale et en respecte parfaitement la forme, noyant quelques extraits de Persepolis sous un flot continu d'ondes suraiguës. Son compatriote Ryoji lkeda, dans sa pièce intitulée $P e r S e$, reste fidèle à sa façon très personnelle de travailler par montage rythmique rapide. La déconstruction de l'œuvre est si complète qu'il n'en subsiste rien de reconnaissable, sinon, peutê̂tre, une intention dans le grain du son. Le codirecteur du projet, Zbigniew Karkowski, intitule sa version Doing By Not Doing et pratique pourtant un bruitisme extrême que I'on dirait à certains moments sur le point de faire éclater la chaîne stéréo. On n'est pas fâché qu'elle soit suivie de la version de la formation américaine Antimatter, qui semble filtrée par un brouillard épais et se développe en un long crescendo jusqu'à un silence abrupte, au centre de la pièce, après quoi s'amorce le mouvement inverse. Avec la pièce Glitche, les Japonais de Construction $\mathrm{Kit}^{26}$ se démarquent et offrent très probablement l'exemple le plus extrême qu'ait produit ce nouveau type de musique ${ }^{27}$; l'œuvre est vraiment un petit bijou en soi, mais le lien avec Xenakis est plus que ténu. Enfin, Francisco Lopez, Laminar, Merzbow et Ulf Langheinrich offrent également des versions très personnelles de l'œuvre, où le lien tient surtout de l'inspiration, plutôt que de la relecture. Comme si l'œuvre avait été transcrite en une partition ne donnant à l'interprète que des indications concernant l'épaisseur des masses sonores, le laissant libre quant à leur agencement.

Est-ce que la manie postmoderne de "faire du neuf avec du vieux " constitue une marque d'essoufflement de l'imagination? Comme plusieurs autres, ce disque, qui
26. Ayant été dans l'impossibilité de trouver la moindre information sur Construction Kit, outre ce qu'en dit le livret, je ne peux que supposer qu'il s'agit d'une formation.

27. On appelle glitch une musique produite à partir de sons parasites que l'électroacousticien moyen considérerait comme des erreurs. 
n'est d'ailleurs que le premier volet d'un diptyque, suggère que non. Chaque composition présente ici a des qualités propres et une originalité qui la distinguent des autres et le regroupement de ces neuf différentes "visions 》 de Persepolis donne une magistrale démonstration de l'éventail des possibles en matière de bruitisme. Otomo Yoshihide ou Zbigniew Karkowski sontils moins légitimés d'aller chercher leur inspiration chez Xenakis que peut l'être, par exemple, un Pascal Dusapin ? Bien sûr que non. L'œuvre du compositeur grec constitue à elle seule un vaste terreau qui pourra inspirer encore longtemps des générations de compositeurs. Les amateurs de sa musique découvriront à coup sûr ici des musiciens intéressants à plus d'un titre, mais ils devront malheureusement faire eux-mêmes les recherches pour trouver sur ces derniers des informations que le livret aurait dû procurer. De même pour ceux qui découvriront Xenakis. C'est là le grand défaut de cette édition. Malgré tout, ceux qui chercheront à se renseigner découvriront, d'un côté comme de l'autre, des univers fascinants.

En conclusion, on est face à trois façons bien différentes d'apprêter l'Histoire. La première est à la limite du révisionnisme, la deuxième est un modèle d'approche documentaire et la dernière opte pour le recyclage créatif. Entre la confusion d'un Kyrou et l'infini que cherche à cerner Sub Rosa, l'hommage à Xenakis d'Asphodel offre un bel exemple, intéressant à tous égards, d'une exploitation éclairée d'une facette de l'héritage fructueux de l'un des plus illustres représentants de la musique du siècle dernier par des artistes qui continuent à défricher la voie à suivre. Dans tous les cas, ils font la preuve, même par la négative, que l'avant-garde ne peut pas faire l'économie de la connaissance de ses sources, au risque d'appliquer l'aphorisme du philosophe américain George Santayana: "Ceux qui ne peuvent se rappeler le passé sont condamnés à le répéter. " N'en déplaise à Dada... 